\title{
PADÉ TABLES OF A CLASS OF ENTIRE FUNCTIONS
}

\author{
D. S. LUBINSKY
}

\begin{abstract}
It is shown that if the Maclaurin series coefficients $\left\{a_{j}\right\}$ of an entire function satisfy a certain explicit condition, then there exists a sequence $\mathscr{S}$ of integers such that $\left[L / M_{l}\right] \rightarrow f$ locally uniformly in $\mathrm{C}$ as $L \rightarrow \infty, L \in \mathscr{S}$, for all nonnegative integer sequences $\left\{M_{L}\right\}_{l=1}^{\infty}$. In particular, this condition is satisfied if the $\left\{a_{j}\right\}$ approach 0 fast enough, or if a subsequence of the $\left\{a_{i}\right\}$ behaves irregularly in a certain sense. Further, the functions satisfying this condition are dense in the space of entire functions with the topology of locally uniform convergence. Consequently, the set of entire functions satisfying the Baker-Gammel-Wills Conjecture is of the second category.
\end{abstract}

1. Introduction. Let $f(z)=\sum_{j=0}^{\infty} a_{j} z^{j}$ be a formal power series and $[L / M]=$ $P_{L M} / Q_{L M}$ be the $L, M$ Padé approximant to $f$ with $Q_{L M}(0)=1$. In 1961 [2, p. 188], Baker, Gammel and Wills conjectured that if $f$ is analytic in $|z| \leqslant 1$, except for a finite number of poles, then a subsequence of $\{[M / M]\}$ converges locally uniformly in (that is, uniformly in compact subsets of) $|z|<1$.

At present there are no counterexamples to this conjecture. In view of a recent paper of Buslaev, Goncar and Suetin [5] on the rows of the Pade table, it seems likely that the conjecture is false in the above form, but possibly true when restricted to entire functions. The most general positive result on pointwise convergence of diagonal Padé sequences is due to Baker [3]. He shows that, for a large class of meromorphic functions with simple poles, at most two subsequences of $\{[N-1 / N]\}$ will produce locally uniform convergence in $\mathbf{C}$, except at poles of $f$. Because of duality theory, this implies convergence of subsequences of $\{[N / N-1]\}$ for a class of entire functions of order $<1$. Diagonal Padé sequences are also known to converge for Stieltjes series [2], Polya frequency series [1] and other special classes [2, 6]. In this note, we prove

THEOREM. Let $f(z)=\sum_{j=0}^{\infty} a_{j} z^{j}$ be entire and transcendental. Assume we are given $K>0$ and nondecreasing positive numbers $\left\{q_{j}\right\}$ such that

$$
\left|a_{L}\right| \leqslant K / \prod_{k=1}^{L} q_{k}, \quad L=0,1,2, \ldots
$$

(We show that there exist $K,\left\{q_{j}\right\}$ satisfying (1) with equality for infinitely many $L$.)

Received by the editors February 2, 1984.

1980 Mathematics Subject Classification. Primary 41A21; Secondary 30E05, 30E10.

Key words and phrases. Padé approximation, entire functions, uniform convergence. 
(a) Assume there exists an infinite sequence $\mathscr{S}$ of positive integers such that

$$
\begin{array}{ll}
q_{L} / q_{L+1} \leqslant 1 / 9, & L \in \mathscr{S}, \\
\left|a_{L}\right|=K / \prod_{k=1}^{L} q_{k}, & L \in \mathscr{S} .
\end{array}
$$

Then $\lim _{L \rightarrow \infty: L \in \mathscr{S}}\left[L / M_{L}\right](z)=f(z)$ locally uniformly in $\mathbf{C}$ for all nonnegative integer sequences $\left\{M_{L}\right\}_{L=1}^{\infty}$. If further,

$$
\lim _{L \rightarrow \infty} M_{L} /\left(q_{L} q_{L+1}\right)^{1 / 2}=0,
$$

then, locally uniformly in $\mathbf{C}, \lim _{L \rightarrow \infty: L \in \mathscr{S}} Q_{L M_{l^{\prime}}}(z)=1$ and $\lim _{L \in \infty ; L \in \mathscr{S}} P_{L M_{L^{\prime}}}(z)=$ $f(z)$.

(b) There exist $K,\left\{q_{L}\right\}, \mathscr{S}$ satisfying (1)-(3) if

$$
\limsup _{L \rightarrow \infty}\left|a_{L}\right|^{1 / L^{2}}=\rho<1 / 3 .
$$

(c) The class of entire functions for which there exist $K,\left\{q_{L}\right\}, \mathscr{S}$ satisfying (1)-(3) is dense in the metric space $\mathscr{A}$ of entire functions equipped with the topology of locally uniform convergence. Consequently the set $\mathscr{B}$ of entire functions satisfying (6) is of the second category in $\mathscr{A}$ :

There exists $\mathscr{S}$ such that $\lim _{L \rightarrow \infty ; L \in \mathscr{S}}\left[L / M_{L}\right](z)=f(z)$ locally uniformly in $\mathbf{C}$ for all sequences $\left\{M_{L}\right\}$ with $0 \leqslant M_{L} \leqslant e^{L}$, $L=1,2, \ldots$

As far as the author can determine, (b) of the theorem is not contained in Baker's result [2], even when $M_{L}=L+1, L=0,1, \ldots$ Further, (c) substantially strengthens Theorem 1 in Borwein [4], where $\mathscr{A}$ and sets of the first and second category were applied for the first time in the context of Padé approximation. We note that $\left\{e^{L}\right\}$ in (6) can be replaced by an arbitrary sequence.

Regarding weaker types of convergence of diagonal sequences, the NuttallPommerenke theorem on convergence in capacity [14] is known to be best possible in various senses $[\mathbf{1 0}, \mathbf{1 1}, \mathbf{1 5}$, as is Wallin's theorem on convergence a.e. [16]. It seems that no one has yet exhibited an entire function of order 2 (or even of finite order) for which $[M / M] \nrightarrow f$ a.e. in $\mathbf{C}$, thereby showing, as is likely, that Edrei's result [7] is best possible. It is noteworthy that the counterexamples in $[10,11,15$, 16], although different in construction, all deal with sparse diagonal sequences like $\left\{\left[2^{M} / 2^{M}\right]\right\}$ and cannot resolve the Baker-Gammel-Wills Conjecture. Other recent results for diagonal sequences appear in $[8,9,12,17]$.

2. Proof of the Theorem. For $L, M=0,1,2, \ldots$, let

$$
V(L / M ; z)=\operatorname{det}\left(\begin{array}{llll}
a_{L} & a_{L+1} & \cdots & a_{L+M} \\
a_{L-1} & a_{L} & \cdots & a_{L+M-1} \\
\vdots & \vdots & & \vdots \\
a_{L-M+1} & a_{L-M+2} & \cdots & a_{L+1} \\
z^{M} & z^{M-1} & \cdots & 1
\end{array}\right)
$$

and $D(L / M)=\operatorname{det}\left(a_{L-i+j}\right)_{i, j=1}^{M}$. 
If $D(L / M) \neq 0$, then $Q_{L M}(z)=V(L / M ; z) / D(L / M)$ is the denominator in $[L / M]$ normalized by $Q_{L M}(0)=1$. The $D(L / M)$ are row rearrangements of the $C(L / M)$ used in [2]. This note is based on the observation that if $\left|a_{L+1}\right|,\left|a_{L+2}\right|, \ldots$ are much smaller than $\left|a_{L}\right|$, then both $D(L / M), V(L / M ; z) \simeq a_{L}^{M}$, and both can be transformed into determinants of matrices with dominant diagonal, to which an inequality of Ostrowski [13] can be applied.

Lemma. Assume (1) and (3). Let

$$
r_{L}= \begin{cases}q_{L} / q_{L+1}, & L=1,2, \ldots, \\ 0, & L \leqslant 0 .\end{cases}
$$

Whenever finite, let

$$
\begin{aligned}
\theta_{L} & =\sum_{j=1}^{\infty} r_{L}^{j / 2}\left\{r_{L+1}^{j-1} r_{L+2}^{j-2} \cdots r_{L+j-2}^{2} r_{L+j-1}\right\}, \\
\mu_{L} & =\sum_{j=1}^{\infty} r_{L}^{j / 2}\left\{r_{L-1}^{j-1} r_{L-2}^{j-2} \cdots r_{L-j+2}^{2} r_{L-j+1}\right\} .
\end{aligned}
$$

Assume

$$
\theta_{L}+\mu_{L} \leqslant 1, \quad L \in \mathscr{S} .
$$

Then for $L \in \mathscr{S}$, and $M=1,2,3, \ldots$,

(a)

$$
2^{-M+1}<\left|D(L / M) a_{L}^{-M}\right|<(3 / 2)^{M-1},
$$

(b)

$$
3^{-2 M+1}<\left|Q_{L M}(z)\right|<3^{M}, \quad|z| \leqslant\left(q_{L} q_{L+1}\right)^{1 / 2} / 2 .
$$

Proof. Let $\xi_{i}=\left(q_{L} q_{L+1}\right)^{i / 2}, i=0, \pm 1, \pm 2, \ldots$.

(a) Multiplying the $i$ th row in $D(L / M)$ by $a_{L}^{-1} \xi_{i}^{-1}$ and multiplying the $j$ th column by $\xi_{j}, i, j=1,2, \ldots, M$, we see

$$
D(L / M) a_{L}^{-M}=\operatorname{det}\left(\frac{a_{L-i+j} \xi_{j}}{a_{L} \xi_{i}}\right)_{i, j}^{M}=\left(b_{i j}\right)_{i, j=1}^{M},
$$

say. Then (1) and (3) yield, for $L \in \mathscr{S}$,

$$
\begin{aligned}
\sum_{j=1: j \neq i}^{M}\left|b_{i j}\right| & \leqslant \xi_{i}^{-1}\left\{\sum_{j=1}^{i-1} \xi_{j} \prod_{k=L-i+j+1}^{L} q_{k}+\sum_{j=i+1}^{M} \xi_{j} \prod_{k=L+1}^{L-i+j} q_{k}^{-1}\right\} \\
& =\sum_{j=1}^{i-1} \xi_{-j} \prod_{k=L-j+1}^{L} q_{k}+\sum_{j=1}^{M-i} \xi_{j} \prod_{k=L+1}^{L+j} q_{k}^{-1} .
\end{aligned}
$$

Now for $k \leqslant L-1, q_{k}=q_{L} r_{L-1} r_{L-2} \cdots r_{k}$ and, for $k \geqslant L+2, q_{k}^{-1}=$ $q_{L+1}^{-1} r_{L+1} r_{L+2} \cdots r_{k-1}$. Since $\xi_{-j} q_{L}^{j}=\xi_{j} q_{L+1}^{j}=r_{L}^{j / 2}$, we see

$$
\begin{aligned}
\sum_{j=1: j \neq i}^{M}\left|b_{i j}\right| & \leqslant \sum_{j=1}^{i-1} r_{L}^{j / 2}\left\{r_{L-1}^{j-1} r_{L-2}^{j-2} \cdots r_{L-j+1}\right\}+\sum_{j=1}^{M-i} r_{L}^{j / 2}\left\{r_{L+1}^{j-1} r_{L+2}^{j-2} \cdots r_{L+j-1}\right\} \\
& <\theta_{L}+\mu_{L} \leqslant 1 .
\end{aligned}
$$


Thus, $\sigma=\max _{i} \sum_{j=1 ; j \neq i}^{M}\left|b_{i j}\right| /\left|b_{i i}\right|<1$. Now, either $\theta_{L} \leqslant 1 / 2$ or $\mu_{L} \leqslant 1 / 2$. Assuming the former is true, apply [13, (8), p. 27] with $n=k=M$ to obtain

$$
\prod_{i=1}^{M-1}\left(1-\sigma \sum_{j=i+1}^{M}\left|b_{i j}\right|\right) \leqslant\left|D(L / M) a_{L}^{-M}\right| \leqslant \prod_{i=1}^{M-1}\left(1+\sigma \sum_{j=i+1}^{M}\left|b_{i j}\right|\right)
$$

and (10) follows, as $\sum_{j=i+1}^{M}\left|b_{i j}\right|<\theta_{L} \leqslant 1 / 2$. When $\mu_{L} \leqslant 1 / 2$, apply [13, (8), p. 27] with $n=M, k=1$.

(b) Now, $Q_{L M}(z)=V(L / M ; z) / D(L / M)$, as $D(L / M) \neq 0$. We estimate $V(L / M ; z)$ as in (a): Multiply the $i$ th row in $V(L / M ; z)$ by $a_{L}^{-1} \xi_{i}^{-1}, i=1,2, \ldots, M$ and multiply the $(M+1)$ th row by $\xi_{M+1}^{-1}$. Further multiply the $j$ th column by $\xi_{j}$, $j=1,2, \ldots, M+1$. Then

$$
V(L / M ; z) a_{L}^{-M}=\operatorname{det}\left(c_{i j}\right)_{i, j=1}^{M+1},
$$

where $c_{i i}=1, i=1,2, \ldots, M+1$ and, for $i=1,2, \ldots, M$,

$$
\sum_{j=1: j \neq i}^{M+1}\left|c_{i j}\right|<\theta_{L}+\mu_{L} \leqslant 1
$$

as before, while for $i=M+1$,

$$
\sum_{j=1 ; j \neq i}^{M+1}\left|c_{i j}\right|=\sum_{j=1}^{M}\left(|z| / \xi_{1}\right)^{M+1-j} \leqslant 1-2^{-M}
$$

if $|z| \leqslant \xi_{1} / 2$. Applying [13, (8), p. 27] with $n=M+1$ and $k=M+1$ if $\theta_{L} \leqslant 1 / 2$ but with $k=1$ if $\mu_{L} \leqslant 1 / 2$, we obtain, for $|z| \leqslant \xi_{1} / 2$,

$$
(1 / 2)^{2 M-1}<\left|V(L / M ; z) a_{L}^{-M}\right|<2(3 / 2)^{M-1} .
$$

Applying (10) yields (11).

Proof of (a) of The Theorem. As $\left\{q_{j}\right\}$ is nondecreasing, $r_{j} \leqslant 1$ for all $j$. Then if $L \in \mathscr{S},(2)$, (7) and (8) imply $\theta_{L}+\mu_{L} \leqslant 1$. Thus (9) holds, and (b) of the Lemma shows $[L / M]$ has no poles in $|z| \leqslant\left(q_{L} q_{L+1}\right)^{1 / 2} / 2$ for all $M \geqslant 0$. Since $f$ is entire, $\lim _{L \rightarrow \infty} q_{L}=\infty$. Using standard techniques [2], the locally uniform convergence of $\left[L / M_{L}\right], L \in \mathscr{S}$, follows. When (4) holds, we note that we can write $Q_{L M_{L}}(z)=$ $\prod_{j=1}^{M^{\prime}}\left(1-z / z_{L M j}\right)$ where $M^{\prime} \leqslant M_{L} ;\left|z_{L M j}\right| \geqslant\left(q_{L} q_{L+1}\right)^{1 / 2} / 2$, and so uniformly in a bounded subset of $\mathbf{C}$, for $L \in \mathscr{S}$,

$$
Q_{L M_{L}}(z)=\left(1+O\left(\left(q_{L} q_{L+1}\right)^{-1 / 2}\right)\right)^{M_{L}}=1+o(1) .
$$

Proof of (b) of The Theorem. We first show that for any transcendental $f$, one can choose $K,\left\{q_{j}\right\}$ satisfying (1) with equality for infinitely many integers. Let

$$
K=\max \left\{1,\left|a_{0}\right|\right\} \text {. }
$$

Define integers $0=j_{0}<j_{1}<j_{2}<\cdots$ and numbers $\left\{q_{j}\right\}$ as follows:

$$
\begin{gathered}
q_{j_{1}}^{-1}=\left(\left|a_{j_{1}}\right| / K\right)^{1 / j_{1}}=\max \left\{\left(\left|a_{j}\right| / K\right)^{1 / j}: j \geqslant 1\right\}, \\
q_{j_{k+1}}^{-1}=\left|a_{j_{k+1}} / a_{j_{k}}\right|^{1 /\left(j_{k+1}-j_{k}\right)}=\max \left\{\left|a_{j} / a_{j_{k}}\right|^{1 /\left(j-j_{k}\right)}: j>j_{k}\right\},
\end{gathered}
$$


$k=1,2,3, \ldots$ If there is more than one choice for some $j_{k}, k \geqslant 1$, we always choose the largest possible value. Define

$$
q_{j}=q_{j_{k+1}}, \quad j_{k}+1 \leqslant j \leqslant j_{k+1}, k=0,1,2, \ldots
$$

It is not hard to see (1) holds, with equality at least if $L \in\left\{j_{1}, j_{2}, j_{3}, \ldots\right\}$. Further by (14), and choice of $j_{k+1}$,

$$
\begin{aligned}
q_{j_{k+1}}^{-1} & =\left|a_{j_{k+1}} / a_{j_{k}}\right|^{1 /\left(j_{k+1}-j_{k}\right)}>\left|a_{j_{k+2}} / a_{j_{k}}\right|^{1 /\left(j_{k+2}-j_{k}\right)} \\
& =\left\{q_{j_{k+2}}^{-\left(j_{k+2}-j_{k+1}\right)} q_{j_{k+1}}^{-\left(j_{k+1}-j_{k}\right)}\right\}^{1 /\left(j_{k+2}-j_{k}\right)} .
\end{aligned}
$$

We deduce

$$
q_{j_{1}}<q_{j_{2}}<q_{j_{3}}<\cdots .
$$

As $f$ is entire, we see $q_{j} \uparrow \infty$ as $j \rightarrow \infty$.

Now let us assume (5). Let $\beta=\limsup _{L \rightarrow \infty} q_{L}^{1 / L}$. Given $\varepsilon>0$, we have for $L \geqslant L_{0}(\varepsilon)$, and some constant $c(\varepsilon)$,

$$
K / \prod_{j=1}^{L} q_{j} \geqslant c(\varepsilon) \prod_{j=1}^{L}(\beta(1+\varepsilon))^{-j}=c(\varepsilon)(\beta(1+\varepsilon))^{-L(L+1) / 2} .
$$

Raising to the power $L^{-2}$ and letting $L \rightarrow \infty$ through $\left\{j_{1}, j_{2}, \ldots\right\}$, we obtain $\rho \geqslant(\beta(1+\varepsilon))^{-1 / 2}$, so that $\beta \geqslant \rho^{-2}$. It follows that $\liminf _{L \rightarrow \infty} q_{L} / q_{L+1} \leqslant \rho^{2}<1 / 9$, and we can choose $\mathscr{S}$ for which (2) holds. But (15) shows that whenever $q_{L}<q_{L+1}$, necessarily $q_{L}=q_{j_{k}}$ for some $k$. Thus (3) also holds.

ProOF OF (c) OF THE TheOREM. For entire $f(z)=\sum_{j=0}^{\infty} a_{j} z^{j}, g(z)=\sum_{j=0}^{\infty} b_{j} z^{j}$, let $d^{*}(f, g)=\max \left\{\left|a_{j}-b_{j}\right|^{1 /(j+1)}: j \geqslant 0\right\}$. This metric is equivalent to that used by Borwein [4], and $\mathscr{A}$ is complete and also a Baire space with respect to $d^{*}$. We first show that the set $\mathscr{C}$ of $f$ for which there exist $K,\left\{q_{j}\right\}, \mathscr{S}$ satisfying (1)-(3) is dense in $\mathscr{A}$. Let $K(f), q_{j}(f), j_{k}(f)$ denote the numbers defined by (12)-(15) for any transcendental $f$. Now fix transcendental $f(z)=\sum_{j=0}^{\infty} a_{j} z^{j}$ and choose $L=j_{n}(f)$, some $n \geqslant 1$. Choose inductively integers $L=L_{0}<L_{1}<L_{2}<\cdots$ such that, for $k=1,2,3, \ldots$,

$$
\left|a_{L_{k+1}} / a_{L_{k}}\right|^{1 /\left(L_{k+1}-L_{k}\right)} \leqslant(1 / 9)\left|a_{L_{k}} / a_{L_{k-1}}\right|^{1 /\left(L_{k}-L_{k-1}\right)} .
$$

Let $g(z)=\sum_{j=0}^{L} a_{j} z^{j}+\sum_{k=1}^{\infty} a_{L_{k}} z^{L_{k}}$. We see from (12)-(15) that $K(g)=K(f)$, $q_{j}(g)=q_{j}(f), j=1,2, \ldots, L$. Further by (17) for $j=1,2, \ldots$ and $k=0,1,2, \ldots$,

$$
\begin{aligned}
\left|a_{L_{k+j}} / a_{L_{k}}\right|^{1 /\left(L_{k+j}-L_{k}\right)} & =\left\{\prod_{l=0}^{j-1}\left|a_{L_{k+1+1}} / a_{L_{k+1}}\right|\right\}^{1 /\left(L_{k+j}-L_{k}\right)} \\
& <\left\{\prod_{l=0}^{j-1}\left|a_{L_{k+1}} / a_{L_{k}}\right|^{\left(L_{k+1+1}-L_{k+1}\right) /\left(L_{k+1}-L_{k}\right)}\right\}^{1 /\left(L_{k+j}-L_{k}\right)} \\
& =\left|a_{L_{k+1}} / a_{L_{k}}\right|^{1 /\left(L_{k+1}-L_{k}\right)} .
\end{aligned}
$$


We deduce $j_{n+k}(g)=L_{k}, \quad k=1,2, \ldots, \quad$ and $q_{L}(g) / q_{L+1}(g) \leqslant 1 / 9, \quad L \in$ $\left\{L_{1}, L_{2}, L_{3}, \ldots\right\}$. Thus $g \in \mathscr{C}$. Further,

$$
d^{*}(f, g) \leqslant \max \left\{\left|a_{j}\right|^{1 /(j+1)}: j>L\right\},
$$

which can be made arbitrarily small by choosing $L$ large enough. Hence $\mathscr{C}$ is dense in the transcendental entire functions and so in $\mathscr{A}$.

Finally, we show, following [4], that the set $\mathscr{B}$ of entire functions satisfying (6) is of the second category. Let $\mathscr{B}_{L, j}=\{f: D(L / M) \neq 0$ and $[L / M]$ has no poles in $\left.|z|<j, \quad M=1,2, \ldots, e^{L}\right\}, L, j=1,2, \ldots$ Let $\mathscr{B}_{k, j}^{*}=\bigcup_{L \geqslant k} \mathscr{B}_{k, j}, k, j=1,2, \ldots$ Clearly, $\mathscr{B} \supset \cap_{j=1}^{\infty} \cap_{k=1}^{\infty} \mathscr{B}_{k, j}^{*}$.

Now, by the Lemma, $\mathscr{C} \subset \mathscr{B}_{k, j}^{*}$ for all $k$ and $j$. Further, as $Q_{L M}(z)$ is continuous in $a_{0}, a_{1}, \ldots, a_{L+M}$ whenever $D(L / M) \neq 0$, we see $\mathscr{B}_{L, j}$ is open for all $L, j$. Hence $\mathscr{B}_{k, j}^{*}$ is open and dense, for all $k, j$. As $\mathscr{A}$ is a Baire space, it follows that $\mathscr{B}$ is of the second category.

REMARK. One can improve on $1 / 3$ in (5) in some cases. Assume

$$
\left|a_{L}\right| \leqslant K\left\{\rho\left(1+\delta_{L}\right)\right\}^{L^{2}}, \quad L=0,1,2, \ldots,
$$

with equality for $L \in \mathscr{S}$, where $\left\{\delta_{L}\right\}$ satisfies

$$
\begin{aligned}
(L+1)^{2} \log \left(1+\delta_{L+1}\right)+(L-1)^{2} \log \left(1+\delta_{L-1}\right)-2 L^{2} \log \left(1+\delta_{L}\right) & \leqslant 0, \\
L & =1,2, \ldots .
\end{aligned}
$$

Set

$$
q_{L}=\left\{\rho\left(1+\delta_{L-1}\right)\right\}^{(L-1)^{2}} /\left\{\rho\left(1+\delta_{L}\right)\right\}^{L^{2}}, \quad L=1,2, \ldots
$$

Then (1), (3) hold, and we see $r_{L} \leqslant \rho^{2}, L=1,2, \ldots$ By (7) and (8),

$$
\theta_{L}+\mu_{L} \leqslant G(\rho)=2 \sum_{j=1}^{\infty} \rho^{j^{2}}, \quad L=1,2, \ldots
$$

Clearly, if $\rho_{0}=0.4559 \cdots$ is the root of $G\left(\rho_{0}\right)=1$, then the conclusions of (a) of the Theorem remain true if $\rho \leqslant \rho_{0}$.

NOTE ADDED IN PROOF. Further results of this type are given in Padé tables of entire functions of very slow and smooth growth, to appear in Constructive Approximation.

\section{REFERENCES}

1. R. J. Arms and A. Edrei, The Padé tables and continued fractions generated by totally positive sequences, Mathematical Essays Dedicated to A. J. Macintyre, Ohio Univ. Press, 1970, pp. 1-21.

2. G. A. Baker, Jr., Essentials of Pade approximants, Academic Press, New York, 1975.

3. Convergence of Pade approximants using the solution of linear functional equations, J. Math. Phys. 16 (1975), 813-822.

4. P. B. Borwein, The usual behaviour of rational approximations, Canad. Math. Bull. 26 (1983), $317-323$.

5. V. I. Buslaev, A. A. Gončar and S. P. Suetin, Convergence of subsequences of the mth row of a Padé table, Mat. Sb. 120 (1983), 540-545. (Russian)

6. A. Edrei, The Pade table of meromorphic functions of small order with negative zeros and positive poles, Rocky Mountain J. Math. 4 (1974), 175-180. 
7. , The Padé table of functions having a finite number of essential singularities, Pacific J. Math. 56 (1975), 429-453.

8. A. A. Gončar and K. N. Lungu, Poles of diagonal Padé approximants and the analytic continuation of functions, Math. USSR.-Sb. 39 (1981), 255-266.

9. E. Hendriksen and H. Van Rossum, Moment methods in Padé approximation, J. Approx. Theory 35 (1983), 250-263.

10. D. S. Lubinsky, Diagonal Padé approximants and capacity, J. Math. Anal. Appl. 78 (1980), 58-67.

11. , Divergence of complex rational approximations, Pacific J. Math. 108 (1983), 141-153.

12. D. S. Lubinsky and A. Sidi, Convergence of linear and nonlinear Pade approximants from series of orthogonal polynomials, Trans. Amer. Math. Soc. 278 (1983), 333-345.

13. A. M. Ostrowski, Note on bounds for determinants with dominant principal diagonal, Proc. Amer. Math. Soc. 3 (1952), 26-30.

14. Ch. Pommerenke, Pade approximants and convergence in capacity, J. Math. Anal. Appl. 41 (1973), $775-780$.

15. E. A. Rahmanov, On the convergence of Pade approximants in classes of holomorphic functions, Math. USSR-Sb. 40 (1981), 149-155.

16. H. Wallin, The convergence of Pade approximants and the size of the power series coefficients, Applicable Anal. 4 (1974), 235-251.

17. Potential theory and approximation of analytic functions by rational interpolation, Proc. Complex Analysis Conf. at Joensuu (I. Laine, O. Lehto, T. Sorvali, Editors), Lecture Notes in Math., Vol. 747, Springer, Berlin, 1979, pp. 434-450.

National Research Institute for Mathematical Sciences of the CSIR, P.O. Box 395, Pretoria 0001, Republic of South Africa 